\title{
Structural Abstraction of Software Verification Conditions ${ }^{\star}$
}

\author{
Domagoj Babić and Alan J. Hu \\ Department of Computer Science \\ University of British Columbia
}

\begin{abstract}
Precise software analysis and verification require tracking the exact path along which a statement is executed (path-sensitivity), the different contexts from which a function is called (context-sensitivity), and the bit-accurate operations performed. Previously, verification with such precision has been considered too inefficient to scale to large software. In this paper, we present a novel approach to solving such verification conditions, based on an automatic abstraction-checking-refinement framework that exploits natural abstraction boundaries present in software. Experimental results show that our approach easily scales to over 200,000 lines of real C/C++ code.
\end{abstract}

\section{Introduction}

Verification conditions (VCs) are logical formulas, constructed from a system and desired correctness properties, such that the validity of verification conditions corresponds to the correctness of the system. Proving validity of verification conditions is an essential step in software verification, and is the focus of this paper.

In general, proving software VCs requires interprocedural analysis, e.g. of the propagation of data-flow facts. Some properties, like proper nesting of lock-unlock calls, tend to be localized to a single function and are amenable to simpler analysis. Many others, especially pointer-related properties, tend to span through many function calls.

To handle the complexity of interprocedural analysis, the software analysis community has developed a number of increasingly expensive abstractions. For instance, path-insensitive analysis does not track the exact path along which a certain statement is executed, while context-insensitive analysis does not differentiate the contexts from which a function is called. These abstractions work well in optimizing compilers, but are not precise enough for verification purposes. Software verification analysis has to be both path- and context-sensitive (*-sensitive) to keep the number of false errors low.

Precise *-sensitive software verification has two components: (1) we need an analysis that takes a piece of software as input and computes VCs as logical formulas in some logic, and (2) once the VCs are computed, we need to check their validity. This paper proposes a novel approach to checking the validity of *-sensitive VCs.

Our approach is an abstraction-checking-refinement framework that exploits the natural function-level abstraction boundaries present in software. Programmers organize code into functions and use them as abstractions. They tend to ignore the details of the

\footnotetext{
* Research supported by a Microsoft Graduate Fellowship and an NSERC Discovery Grant.
} 
effects of the function on the caller's context - the easiest invariant to remember is to remember no invariant at all. Analogously, our approach initially treats individual effects of a function call as unconstrained variables and incrementally adds constraints corresponding to the effects of the function call. We demonstrate that such a structural refinement approach works well, even on large general-purpose $\mathrm{C} / \mathrm{C}++$ applications.

\subsection{Related Work}

Interprocedural analysis can have many forms, and is commonly based on some form of summarization. Usually, the more expressive the summaries are, the higher the computational complexity. For instance, if the set of data-flow facts is a finite set, and the data-flow functions distribute over the confluence, interprocedural data-flow analysis can be done in polynomial time [21]. If the summaries are composed of predicates over arbitrary logic the analysis gets more complex, depending on the underlying logic.

If the number of predicates is relatively small, predicate abstraction [14] makes it possible to represent summaries compactly as BDDs [5]. This approach has been effectively used in SLAM [3] and BLAST [15[16]. Predicate abstraction is very coarse, and hasn't been shown to scale well to large applications for data-intensive properties. Its advantage is that the VCs given to the theorem prover are relatively simple, corresponding to a conjunction of conditions on some path in the program. Saturn [25] handles lock-properties in a similar way - by computing summaries as projections onto a set of predicates, with the difference that it does not abstract VCs before passing them to the theorem prover. In contrast to the above-mentioned approaches, the technique presented in this paper allows summaries to be arbitrary expressions, rather than just projections onto a set of predicates.

Livshits and Lam [20] proposed a path- and context-sensitive points-to analysis and used it for simple security checks. Their summaries represent definition-use chains required for tracking pointers interprocedurally. They demonstrated their analysis on small programs up to 13 thousand lines of code. Whaley and Lam [23] stressed the importance of context-sensitive analysis and proposed a brute force approach to contextsensitive, inclusion-based pointer alias analysis. Their analysis, implemented in the bddbdd system, represents input-output relations as BDDs [5]. The BDD-based approach seems to work well for tracking a set of locations, but it is not applicable to verification of assertions because BDDs are known to suffer from exponential blow-up on multiplication, division, and barrel shifters - all frequent operations in software. Both works focused on the software analysis side, while our focus is on proving *-sensitive VCs. We believe that our results could improve the scalability of their approach.

The CBMC tool [76] verifies C programs, to bounded depth, with bit-accuracy and *-sensitivity. The approach is direct symbolic execution of the $\mathrm{C}$ into a SAT instance, unrolling all loops and inlining all function calls, so solving the generated VC is the performance bottleneck for large software. Our results address that bottleneck.

In the domain of programs limited to static memory allocation, Astrée [4] has been successfully applied to verification of mission-critical software systems. Although context-sensitive over the chosen abstract domain(s), Astrée was designed for systems that contain no goto statements, no dynamic memory allocation, no recursive calls, no 
recursive data structures, and no pointer arithmetic. Since our focus is verification of assertions in general purpose software, these constraints were not acceptable.

Context-sensitivity is only one component of the problem. Path-sensitivity is the other. The BLAST and SLAM software model checkers enumerate paths one-by-one, hoping that refinement will refute many paths with each added predicate. For each path, the model checker constructs an abstracted theorem prover query, which can correspond to a path that spans through many functions. Such path enumeration during the abstraction-checking-refinement loop seems wasteful — SAT solvers are extremely efficient in path enumeration and refutation of infeasible paths, so we believe that path enumeration should be left to the SAT solver.

Others have realized the importance of letting the theorem prover enumerate the paths as well. For instance, software verification systems like Boogie [18] and ESC/Java [12] do construct a single formula and let the theorem prover enumerate the paths. However, these systems rely on the user to provide interface abstractions, and do not attempt to abstract the formulas before calling the theorem prover.

Our approach to proving *-sensitive VCs merges both SAT-solver-based path enumeration and abstraction, yielding a precise, but practically efficient alternative to previous methods.

\section{A Review of Verification Condition Generation}

Traditionally, VCs are computed by Dijkstra's weakest precondition transformer [10], as is done for example in ESC/Java [12] and Boogie [18]. A naïve representation of VCs computed by the weakest precondition can be exponential in the size of the code fragment being checked, but this blow-up can be avoided by the introduction of fresh variables to represent intermediate expressions [22 13,19]. Here, we give a quick overview of weakest-precondition-based VC computation to illustrate the process, some common problems, and an efficient representation.

Consider the following simple program (modified from [19]):

$$
\begin{aligned}
& \mathrm{S} 1: \text { if }(\mathrm{x}<0)\{\mathrm{y}=-2 * \mathrm{x}-\mathrm{y} ;\} \\
& \mathrm{S} 2: \mathrm{y}=\mathrm{x}+\mathrm{y} ; \\
& \mathrm{S} 3: \text { assert }(0<=\mathrm{y})
\end{aligned}
$$

The VC can be computed as the weakest liberal precondition wlp() of a sequential composition of those three statements with respect to true, giving:

$$
\begin{aligned}
\operatorname{wlp}(S 1 ; S 2 ; S 3, \text { true }) & =\operatorname{wlp}(S 1, w \operatorname{wlp}(S 2, \text { wlp }(S 3, \text { true }))) \\
& =\operatorname{wlp}(S 1, w \operatorname{wl}(S 2,0 \leq y)) \\
& =\operatorname{wlp}(S 1,0 \leq x+y) \\
& =0 \leq \operatorname{ITE}(x<0,-(x+y), x+y)
\end{aligned}
$$

where ITE is the if-then-else operator. Obviously, continuous application of wlp () can lead to exponential blowup in the size of the formula. To avoid the blowup, we can perform renaming, which guarantees a single point of definition for each variable (as in Single Static Assignment (SSA) form [9]): 


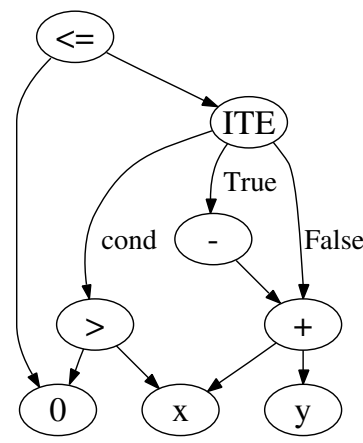

Fig. 1. Graph Representation of the Verification Condition. Non-leaf nodes are labeled with operators; leaf nodes, with variables and constants. Operator nodes are connected to their operands by edges.

$\mathrm{S} 1:$ if $(\mathrm{x} 0<0)\{\mathrm{y} 1=-2 * \mathrm{x} 0-\mathrm{y} 0 ;\}$ else $\{\mathrm{y} 1=\mathrm{y} 0 ;\}$

S2: $\mathrm{y} 2=\mathrm{x} 0+\mathrm{y} 1$;

S3: assert $(0<=$ y2 $)$;

Since each variable has a single point of definition, assignments can be replaced with equivalences (passive commands in [19]), and then wlp $\left(S 1 ; S 2 ; S 3,0 \leq y_{2}\right)$ boils down to:

$$
\left(x_{0}<0 \Rightarrow\left(y 1 \equiv-2 x_{0}-y_{0}\right)\right) \wedge\left(x_{0} \geq 0 \Rightarrow\left(y_{1} \equiv y_{0}\right)\right) \wedge\left(y_{2} \equiv x_{0}+y_{1}\right) \wedge\left(0 \leq y_{2}\right)
$$

Exponential blowup is avoided at the expense of introduction of fresh variables.

The same VC can be represented in the form of a graph. In particular, we simply represent a logical formula as a directed, acyclic graph, in which non-leaf nodes are labeled with operators, their children are their operands, and the leaves are labeled with variables or constants. A graph representation of a logical formula such that all common subexpression nodes have been merged will be called a maximally-shared graph. Figure 1 depicts a maximally shared graph representation of the computed VC in Eq. 4. The advantage of using maximally-shared graphs for VC representation is that the elimination of common subexpressions is simple, while the graph is still linear in the size of the code fragment.

The work in this paper is to support our static checker CALYSTO, which is being designed to be a general-purpose, bit-precise assertion checker. CALYSTO implements an efficient interprocedural symbolic execution algorithm [1] that converts SSA (computed using the LLVM compiler framework [17]) into function summaries and VCs in the form of acyclic maximally-shared graphs. For each location that a function modifies, CAlysto computes the resulting expression in terms of the function inputs (including globals). Each such expression is represented as a separate summary expression, which gives fine-grained control during the refinement process (Sec. 3.2). Like other static checkers, CALYSTO makes a few unsound approximations. For example, loops are unrolled a fixed number of times, with the additional assumption that the loop test fails at the loop exit, as is done in ESC/Java [12], Saturn [25], and older versions of 
Boogie [18]. We could also handle loops soundly by using loop invariants (computed by any technique), as is done in Boogie. Similarly, CALYSTO handles non-constant array indices by unsoundly replacing them with constant indices. In addition, $\mathrm{CA}_{\text {A }}$ LYSTO makes the unsound assumption that pointers passed as function parameters are not aliased, as in [2025]. However, CALYSTO's computed VCs are *-sensitive, fully bit-accurate, and support all standard operators (e.g., signed/unsigned division and multiplication on bit-vectors, etc.), except that floating-point arithmetic is not yet implemented.

\section{Exploiting Natural Abstraction Boundaries}

We begin with an example that provides intuition about how our approach solves * sensitive VCs. The code used in the example is a simplified and slightly modified piece of code from a real application 1 To prove an assertion, we need to prove either that the assertion itself is unreachable, or that it always evaluates to true. Through the example, we shall follow a sequence of steps needed to prove the assertion on line 22 .

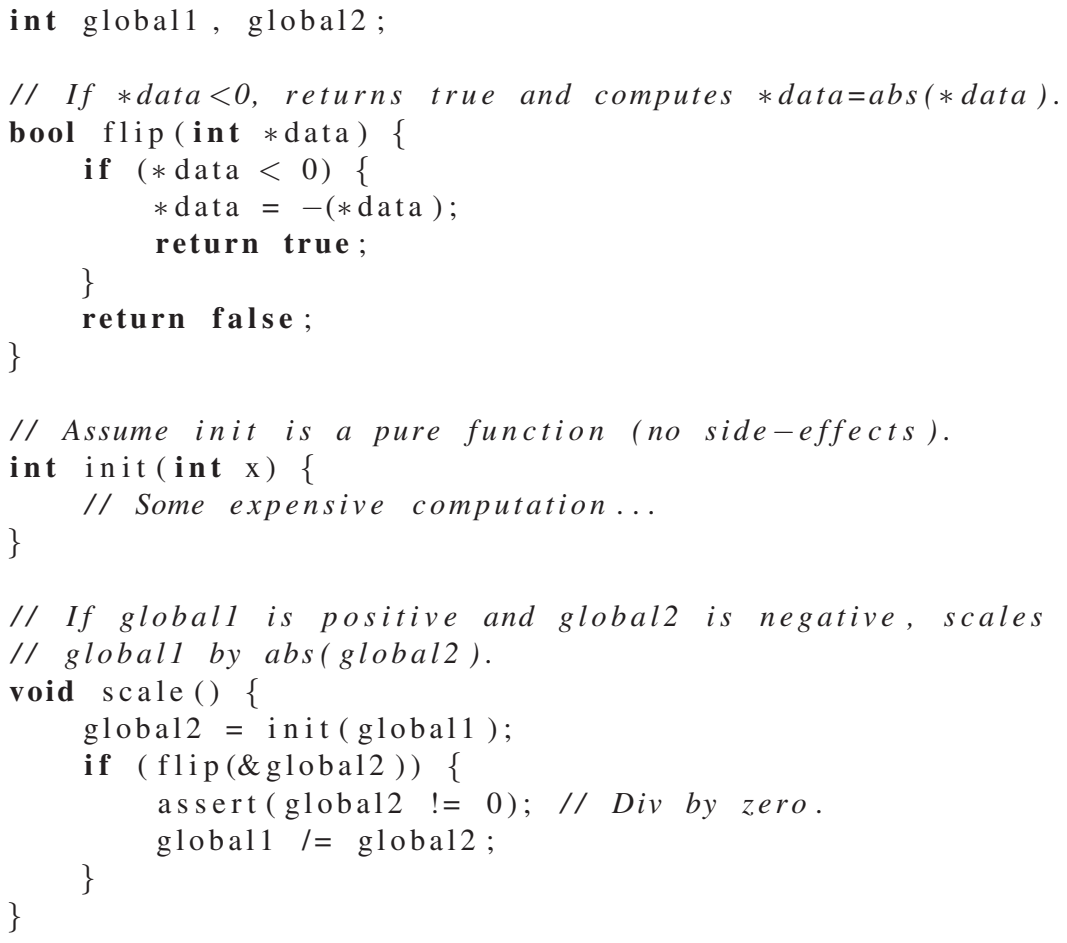

\footnotetext{
${ }^{1}$ The example is modified from our modular arithmetic theorem prover SPEAR.
} 
(a)

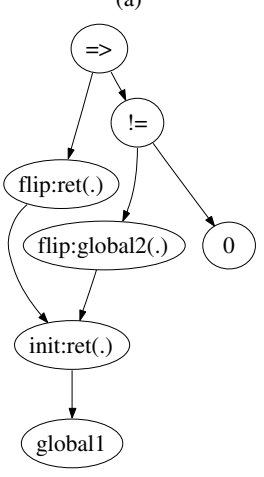

(b)

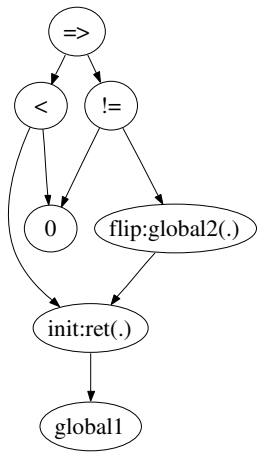

(c)

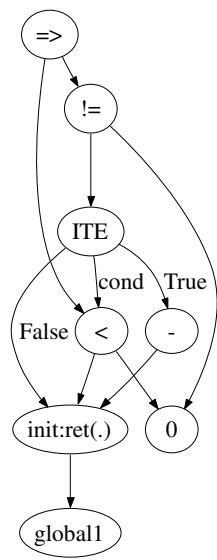

(d)

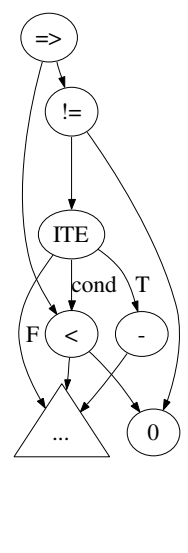

Fig. 2. Sequence of Refinements of the Computed VC. Summary nodes are structurally refined in the following sequence: flip:ret, flip:global2, and finally init:ret. The subgraph obtained by the refinement of init:ret is represented by a triangle. For simplicity, these figures do not show pointer references and dereferences.

As mentioned earlier, the symbolic execution will compute a graph representing each effect of each function in terms of its parameters (and globals). For example, the function $\mathrm{flip}$ has two effects: a boolean return value and its effect on the location pointed to by its parameter. At the caller's side, the symbolic execution initially denotes effects of a function call by a placeholder operator node. For example, the return value of a call to flip will be an operator node labeled flip : ret whose child is the argument to flip.

The VC will be an implication: if line 22 is reachable, then the asserted condition must hold. Let us ponder the structure of the computed VC. The antecedent contains two nested function calls. The consequent is a simple comparison of zero with the effect of flip on the global variable. Observe that the expression is written in terms of the initial values of all involved variables, facilitating common subexpression elimination by simple graph rewriting. Graphically, the VC can be represented as a maximallyshared graph (Fig.2a). Summaries of the individual effects of each called function are at first represented as unconstrained fresh variables. Those nodes will be called summary nodes. Interpretation of a summary node corresponds to replacing the node with a node that represents the summarized expression. Such expansion corresponds to a round of inlining.

To be fully context-sensitive, the obvious approach is to completely inline all calls. Such inlining leads to exponential blow-up even on small applications. We found that aggressive inlining of non-recursive function calls works only on several very small applications, resulting in roughly 50-180X increase in the size of the code.

A better approach is to track the individual effects of a function separately. This finegrained approach makes it possible to expand only the slice of the called function that is actually in the cone of influence of the verified property. We consider this approach to be 
the state of the art and shall use it as the base case for comparison with our abstractionbased approach in Sec. 4. Together with the common subexpression elimination, this approach is more scalable, but does not offer satisfactory performance.

The crux of the problem is that interprocedural analysis can't decide when to stop inlining. After only three refinements, ${ }^{*}$-sensitive analysis would expand computationally expensive init, rendering the problem much harder for the decision procedure. However, the VC can be proved to be valid after only two refinements

$$
\begin{aligned}
& \text { Let } x=\text { init }: \text { ret }(\text { global } 1) \\
& x<0 \Rightarrow \operatorname{ITE}(x<0,-x, x) \neq 0
\end{aligned}
$$

which simplifies to true, no matter what init returns. Cases like this appear frequently in practice, especially during *-sensitive verification of data-intensive properties, like checking of assertions or global pointer properties.

Our approach gradually refines the maximally-shared graph, until the VC becomes valid, or the decision procedure finds a falsifying assignment that does not depend on any summary nodes. The rest of this section gives the details of our approach.

\subsection{Algorithm Overview}

The proposed approach follows the general paradigm of automatic, counter-exampleguided, abstraction refinement [8], but unlike typical CEGAR approaches, our abstraction and refinement operations are entirely structural, and the refinement works incrementally on abstract counterexamples (rather than concretizing the abstract counterexample, proving it spurious, and then analyzing the proof). Locations modified by a function call (either indirectly through a pointer, or directly via returned values) are initially considered to be unconstrained variables. Those unconstrained variables are incrementally refined until the formula represented by the graph becomes valid, or the falsifying assignment does not depend on any unconstrained variables. In our case, incremental refinement is structural refinement on the maximally-shared graph. The refinement step replaces an unconstrained variable with a subgraph that represents the summary expression and the edges that were pointing to the unconstrained variable are relinked to point to the newly constructed expression. We shall say that refinement expands summary nodes.

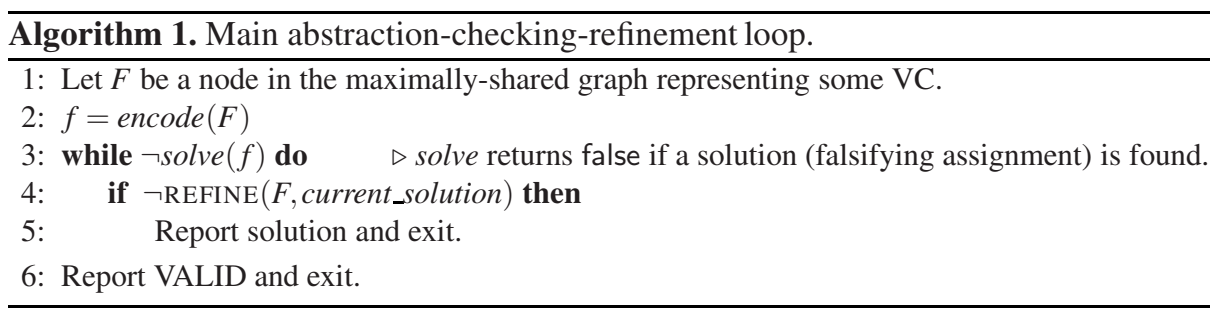

An abstract rendition of our algorithm is given in Alg. 1. The checked verification condition is represented by a root $F$ in the maximally-shared graph. The algorithm encodes $F$ on the fly into formula $f$ and passes it to the decision procedure $(\operatorname{solve}())$. In 
our case, $F$ is bit-accurately translated to CNF by the standard Tseitin transform [22], but from the maximally shared graph after common subexpressions have been eliminated. Summary nodes are encoded as unconstrained variables. If the decision procedure proves $f$ valid, we are done. Otherwise, refinement takes $F$ and the table of current assignments to variables represented by nodes in the support of $F$, and returns true if the graph was refined, and false otherwise. If the graph was not refined, then all the summary nodes related to the falsifying assignment have been expanded, and the main loop terminates. Otherwise, the abstraction-checking-refinement cycle continues. Since maximally-shared graphs are acyclic, the algorithm necessarily terminates.

The algorithm interacts gracefully with incremental decision procedures - each expansion of a summary node replaces only a single node with the expression represented by the summary node, monotonically increasing the set of constraints.

Our lazy approach to interpretation of function summaries resembles the intuition behind lazy proof explication [11], a technique used to bridge between different theories in a theorem prover. The shared intuition is to abstract away expensive reasoning - expanding a function summary or solving a sub-theory query - as unconstrained variables, and then constrain them lazily, only as needed to refute solutions to the abstracted problem. The specifics of what to abstract and how to refine, of course, are different, since we are solving different problems.

Since critical software bugs (e.g. [2]) are often caused by the finite nature of bitvector arithmetic, it is important to maintain the bit-level behavior of the verified software. CALYSTO computes bit-precise VCs, which are translated to CNF directly even expensive 64-bit arithmetic operations, like division and remainder, are handled precisely. The bit-vectors are represented with the same bit-width as in the compiled code. In our case that means that integers and pointers are represented with 64 bits.

Path enumeration is completely left to the SAT solver. We found that it is important for the SAT solver to process the variables in an order that roughly corresponds to reverse preorder traversal (all predecessors are visited before the successors). If the opposite traversal is used, the solving phase typically requires $7-10 \mathrm{X}$ more time. This supports our conclusion that most of the paths become infeasible close to the VC root node.

\subsection{Structural Refinement}

The first few iterations of the main loop of Alg. 1 will likely return false counterexamples, since the initial abstraction is usually very crude. So, the refinement algorithm has to identify very quickly a set of summary nodes that are relevant to the found solution.

The algorithm attempts to minimize the number of expanded summaries to avoid expensive computation. Given a falsifying assignment, our refinement scheme searches the graph and selects a single summary node to expand, thereby refining the model. In particular, the algorithm starts traversing the formula from the VC root. During the traversal, the algorithm detects don't-care values - values that are irrelevant to the current solution and can therefore be ignored 2 To formalize the concept of don't-care values, we define absorptive element as:

\footnotetext{
2 The anonymous reviewers noted a connection between our analysis and strictness analysis in functional programming, as well as the work of Wilson and Dill [24]. The commonality is
} 
Definition 1 (Absorptive Element). If there exists an element a for some operator $\star$, such that $\forall x: a \star x=a$, then $a$ is an absorptive element of $\star$, denoted as abelem $(\star)=a$.

For instance, abelem $(\wedge)=$ false, abelem $(*)=0$, and so on.

If the decision procedure returns a falsifying assignment, each node $F$ in the graph representing the checked VC has some assigned value, which we shall denote as val $(F)$. If $F$ is an operator $\star$, our algorithm checks val $(x)$ for each operand $x$ of $F$. If val $(x)$ is an absorptive element of $\star$, it is a sufficient explanation of the value of $F$ in the falsifying assignment (the other operand is a don't-care). Hence, it suffices to refine only $x$. Our refinement procedure is given in Alg. 2. As is usual for graph traversal, visited nodes are marked during traversal to avoid re-visiting nodes; marking is not shown in the pseudocode.

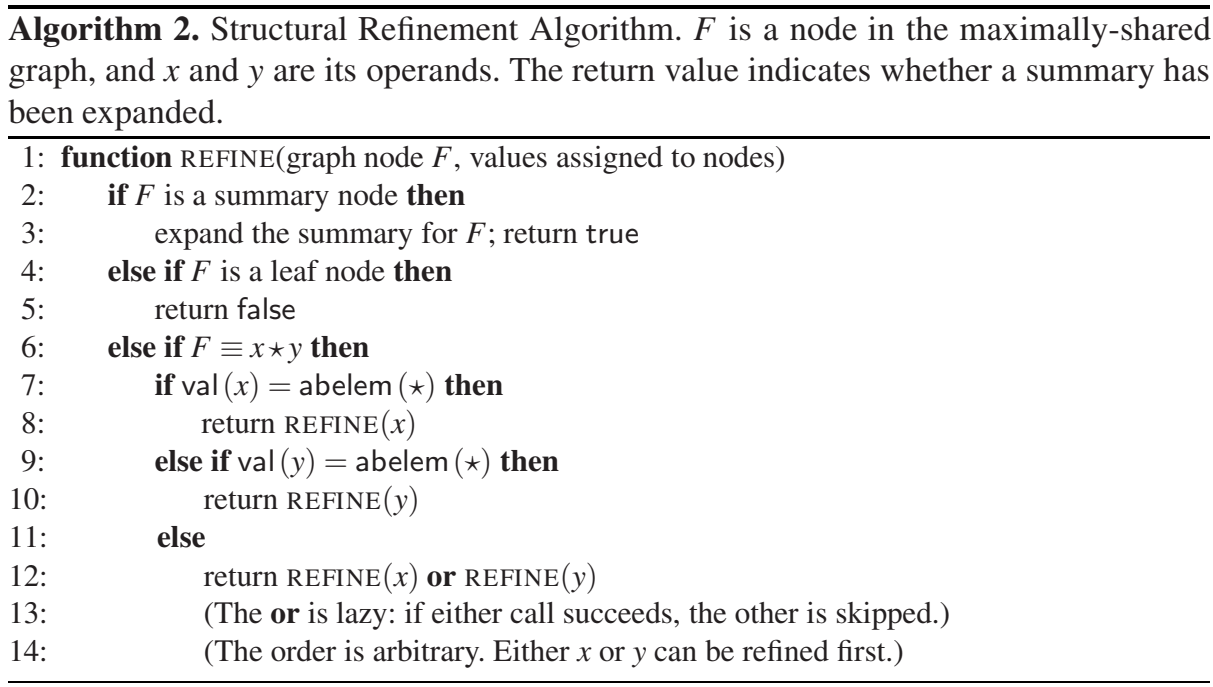

Some operators (like implication and if-then-else) do not have absorptive elements, but allow similar don't-care analysis. Our implementation performs such reductions according to the rules in Alg. 3.

Returning to the example in Fig. 2] in 2a, the checker treats the placeholder nodes as unconstrained variables and finds a falsifying assignment where flip : ret is true and the $!=$ is false. Alg. 3 will derive the refinement in $2 \mathrm{~b}$, where a possible falsifying solution gives the init : ret node a negative value. Next, the algorithm might choose to expand the flip : global2 node, yielding the refinement in 2 k, which is valid. We were able to avoid the expensive expansion of the init : ret node.

the goal of finding cases in which a value is not used or needed. For example, in an ITE in a functional programming language, the condition argument is strict because it is always evaluated, whereas the other two arguments are non-strict. In our case, we are refining a falsifying solution, so we have much more don't-care information available, e.g., we know the value of the condition argument, so we know exactly which branch need not be refined. 


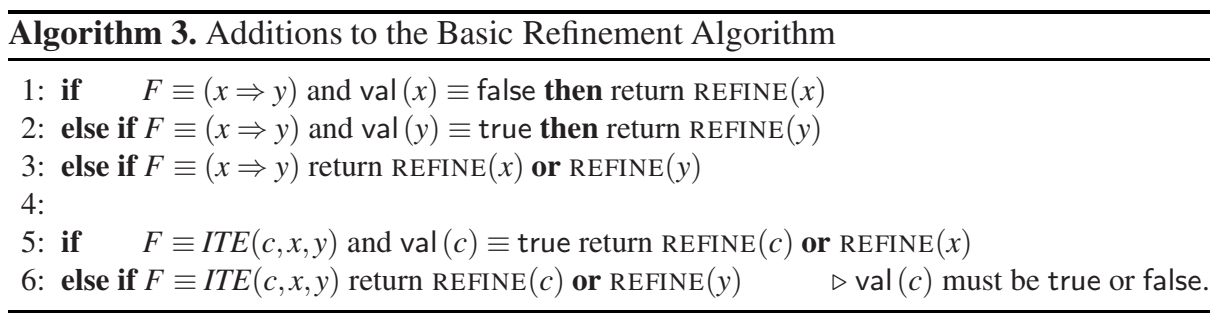

Unlike other approaches, our approach to refinement does not require a theorem prover. The downside is that our refinement might be less precise and result in more refinement cycles. However, each refinement cycle only adds additional constraints to the decision procedure incrementally, making the solving phase more efficient as well.

\section{Experimental Results}

To test our approach, we used CALYSTO to generate VCs for six real-world, publiclyavailable $\mathrm{C} / \mathrm{C}++$ applications, ranging in size from 9 to 228 thousand lines of code (KLOC) before preprocessing. The benchmarks are the Dspam spam filter, our boolean satisfiability solver HYPERSAT, the Licq ICQ chat client, the OpenLDAP implementation of the Lightweight Directory Access Protocol, the Wine Windows OS emulator, and the Xchat IRC client. For each program, for each pointer dereference, we generated a VC to check that the pointer is non-NULL (omitting VCs that were solved trivially by our expression simplifier).

CALYSTO has a simple, non-recursive expression simplifier that runs during symbolic execution. The simplifier rules are numerous, but straightforward. We noticed that performing constant propagation during the simplification reduces the memory footprint, but does not drastically speed-up the solving phase because our modular arithmetic theorem prover SPEAR (like many others) performs aggressive constant propagation on its own. Other, slightly more complex rules, like $\operatorname{ITE}(c, x, \neg c \wedge y) \equiv$ $\operatorname{ITE}(c, x, y)$ do speed up the solving phase, but not drastically.

As the basis for comparison, we also solved the VCs using eager expansion of sliced summaries (described in Sec. 3). The approaches were tested under equal conditions: the same simplification and common subexpression elimination were applied to both approaches after every summary expansion, before calling the SAT solver. The same SAT solver was used for both the base case and our approach.

Table 1 and Fig. 3 summarize the results. In a large majority of cases, the structural abstraction approach is superior to the eager approach, which suffers $81 \%$ more timeouts, and $75 \%$ longer runtime. There are some cases, however, where the eager approach performs significantly better. Analyzing those cases, we found that occasionally our simplifier can simplify some expanded summaries to trivial constants, which in turn can make the VC trivially easy to solve. For example, the most frequent case we have seen is when an expanded summary, which is an antecedent in an implication, trivially simplifies to false, rendering the whole implication true. A priori, there is no way to know whether or not an expanded summary will drastically simplify the VC 
Table 1. The first column gives the name and version of the benchmark, KLOC is the number of source code lines (in thousands) before preprocessing, and \#VCs is the number of checked VCs. The next four columns give the total run time in seconds (including timeouts) and the number of timeouts, for the base approach and for our new structural abstraction and refinement method. The timeout limit was 300 seconds. Experiments were performed on a dual-processor AMD X2 4600+ machine with 2 GB RAM, running Linux 2.6.15.

\begin{tabular}{|l|r|r||r||r||r|r|}
\hline \multirow{2}{*}{ Benchmark } & KLOC & \multirow{2}{*}{ \#VCs } & \multicolumn{2}{|c||}{ Base Approach } & with Struct. Abs./Ref. \\
\cline { 4 - 7 } & & & Time $(\mathrm{sec})$ & Timeouts & Time $(\mathrm{sec})$ & Timeouts \\
\hline \hline Dspam v3.6.5 & 37 & 8003 & 4451 & 12 & 3758 & 10 \\
\hline HyperSAT v1.7 & 9 & 427 & 32602 & 108 & 27025 & 81 \\
\hline Licq v1.3.4 & 20 & 5165 & 24103 & 50 & 4072 & 4 \\
\hline OpenLDAP v2.3.30 & 228 & 4935 & 738 & 0 & 572 & 0 \\
\hline Wine v0.9.27 & 126 & 8831 & 2598 & 0 & 2145 & 0 \\
\hline Xchat v2.6.8 & 76 & 24045 & 18914 & 13 & 10024 & 6 \\
\hline \hline Total & 496 & 55583 & 83406 & 183 & 47596 & 101 \\
\hline
\end{tabular}

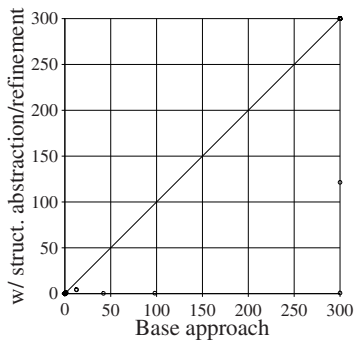

(a) Dspam

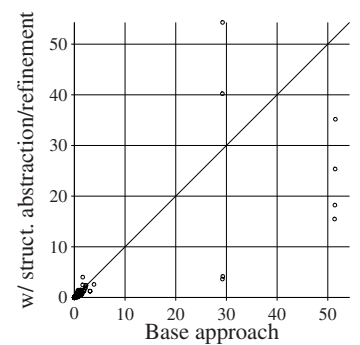

(d) OpenLDAP

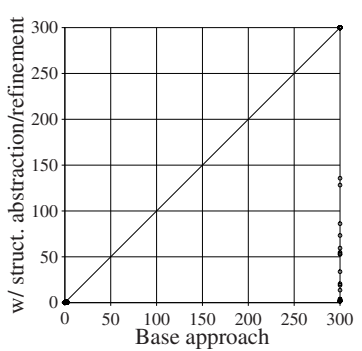

(b) HyperSAT

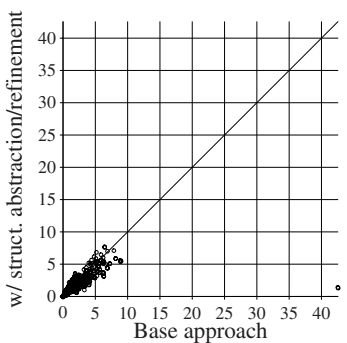

(e) Wine

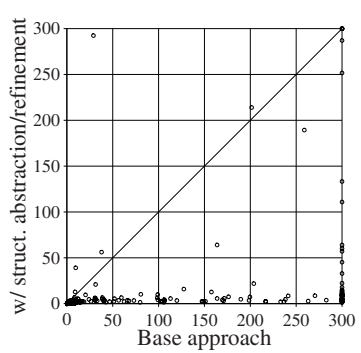

(c) Licq

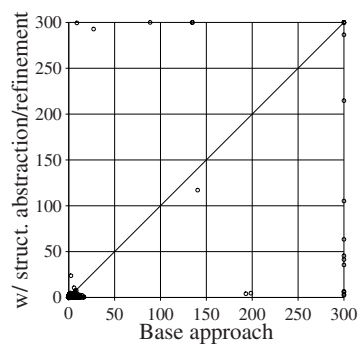

(f) Xchat

Fig. 3. Results presented as scatter plots. Timeouts are plotted at 300secs.

(akin to the classic debate of eager vs. lazy constraint propagation in SMT solvers). Our experimental results, though, show that for solving software VCs, laziness wins.

Overall, our structural abstraction-checking-refinement approach is able to quickly verify *-sensitive, bit-accurate VCs from large software. Moving forward, we believe there is additional structure to be exploited, and that is the direction of future work. 


\section{Discussion}

We believe there are two main reasons behind the success of our approach: efficient path enumeration and exploiting natural abstraction boundaries in software.

In our experience, path enumeration dominates the cost of software verification, even when the loops are abstracted away. In the worst case, any path-sensitive analysis has to analyze all the paths. We use our SAT-solver-based prover SPEAR to enumerate paths efficiently, but the order in which any SAT solver processes constraints is important. Most solvers add variables to the decision queue in the order in which the variables are found in the clauses. So, by starting the SAT solver from a root of the VC graph and letting it enumerate paths, we explore paths in a breadth-first manner. In analyzing several opensource applications, we realized that most paths in an average program are infeasible, and this breadth-first path exploration prunes more paths more quickly. Contemporary software model checkers do not exploit this fact, but rather rely on depth-first search, performing numerous calls to the theorem prover through a counterexample-driven refinement process, just to refute a path that is most likely to be infeasible anyway. Similarly, the lazy summary expansion prunes obviously infeasible paths before function summaries are expanded and analyzed, decreasing the number of paths that need to be explored even further.

The proposed abstraction-refinement approach exploits the natural abstraction boundaries in software that correspond closely to the programmer's mental model. To manage complexity, programmers tend to organize code into structural units (functions) and use them as abstractions - whatever a function returns, the rest of the code should work. This common programming tactic, which reduces the mental load on the programmer, inspired our approach. To the extent that programmers use functions effectively as abstractions, our refinement algorithm can avoid expanding the details that were abstracted away.

Our simple abstraction does not rely on the standard abstraction domains. Hence, the contributions of this paper can be applied to a wide range of software analysis tools that require interprocedural analysis and a decision procedure. If further complexity reduction is needed, we believe our approach should be compatible with classical abstract domains and other abstraction techniques.

\section{References}

1. Babić, D., Hu, A.: Fast Symbolic Execution for Static Checking (submitted for publication)

2. Babić, D., Musuvathi, M.: Modular Arithmetic Decision Procedure. Technical Report TR2005-114, Microsoft Research Redmond (2005)

3. Ball, T., Majumdar, R., Millstein, T., Rajamani, S.: Automatic Predicate Abstraction of C Programs. Programming Language Design and Implementation, pp. 203-213 (2001)

4. Blanchet, B., Cousot, P., Cousot, R., Feret, J., Mauborgne, L., Miné, A., Monniaux, D., Rival, X.: A static analyzer for large safety-critical software. Programming Language Design and Implementation, pp. 196-207 (2003)

5. Bryant, R.: Graph-based algorithms for boolean function manipulation. IEEE Trans. Comput. 35(8), 677-691 (1986) 
6. Clarke, E., Kroening, D., Lerda, F.: A tool for checking ANSI-C programs. In: Jensen, K., Podelski, A. (eds.) TACAS 2004. LNCS, vol. 2988, pp. 168-176. Springer, Heidelberg (2004)

7. Clarke, E., Kroening, D., Yorav, K.: Behavioral consistency of $\mathrm{C}$ and Verilog programs using bounded model checking. In: Design Automation Conference, pp. 368-371 (2003)

8. Clarke, E.M., Grumberg, O., Jha, S., Lu, Y., Veith, H.: Counterexample-guided abstraction refinement. In: Emerson, E.A., Sistla, A.P. (eds.) CAV 2000. LNCS, vol. 1855, pp. 154-169. Springer, Heidelberg (2000)

9. Cytron, R., Ferrante, J., Rosen, B.K., Wegman, M.N., Zadeck, F.K.: Efficiently Computing Static Single Assignment Form and the Control Dependence Graph. ACM Trans Programming Languages and Systems 13(4), 451-490 (1991)

10. Dijkstra, E.W., Scholten, C.S. (eds.): Predicate Calculus and Program Semantics. Springer, Heidelberg (1990)

11. Flanagan, C., Joshi, R., Ou, X., Saxe, J.B.: Theorem proving using lazy proof explication. In: Hunt Jr., W.A., Somenzi, F. (eds.) CAV 2003. LNCS, vol. 2725, pp. 355-367. Springer, Heidelberg (2003)

12. Flanagan, C., Leino, K.R.M., Lillibridge, M., Nelson, G., Saxe, J.B., Stata, R.: Extended static checking for Java. Programming Language Design and Implementation, pp. 234-245 (2002)

13. Flanagan, C., Saxe, J.B.: Avoiding exponential explosion: generating compact verification conditions. Principles of Programming Languages, pp. 193-205 (2001)

14. Graf, S., Saidi, H.: Construction of abstract state graphs with PVS. In: Grumberg, O. (ed.) CAV 1997. LNCS, vol. 1254, pp. 72-83. Springer, Heidelberg (1997)

15. Henzinger, T., Jhala, R., Majumdar, R., Sutre, G.: Lazy Abstraction. Principles of Programming Languages, pp. 58-70 (2002)

16. Henzinger, T.A., Jhala, R., Majumdar, R., McMillan, K.L.: Abstractions from proofs. Principles of Programming Languages, pp. 232-244 (2004)

17. Lattner, C., Adve, V.: LLVM: A Compilation Framework for Lifelong Program Analysis \& Transformation. In: CGO '04: Proceedings of the International Symposium on Code Generation and Optimization, p. 75. IEEE Computer Society, Washington, DC, USA (2004)

18. Leino, K.R.M., Müller, P.: A verification methodology for model fields. In: Sestoft, P. (ed.) ESOP 2006 and ETAPS 2006. LNCS, vol. 3924, pp. 115-130. Springer, Heidelberg (2006)

19. Leino, K.R.M.: Efficient weakest preconditions. Inf. Process. Lett. 93(6), 281-288 (2005)

20. Livshits, V.B., Lam, M.S.: Tracking Pointers with Path and Context Sensitivity for Bug Detection in C Programs. In: European Software Engineering Conference/International Symposium on Foundations of Software Engineering, pp. 317-326 (2003)

21. Reps, T., Horwitz, S., Sagiv, M.: Precise interprocedural dataflow analysis via graph reachability. Principles of Programming Languages, pp. 49-61 (1995)

22. Tseitin, G.S.: On the complexity of derivation in propositional calculus. In: Siekmann, J., Wrightson, G. (eds.) Automation of Reasoning 2: Classical Papers on Computational Logic 1967-1970, pp. 466-483. Springer, Heidelberg (1983)

23. Whaley, J., Lam, M.S.: Cloning-based context-sensitive pointer alias analysis using binary decision diagrams. Programming Language Design and Implementation, pp. 131-144 (2004)

24. Wilson, C., Dill, D.L.: Reliable verification using symbolic simulation with scalar values. In: 37th Design Automation Conference, pp. 124-129. ACM/IEEE (2000)

25. Xie, Y., Aiken, A.: Scalable error detection using boolean satisfiability. Principles of Programming Languages, pp. 351-363 (2005) 